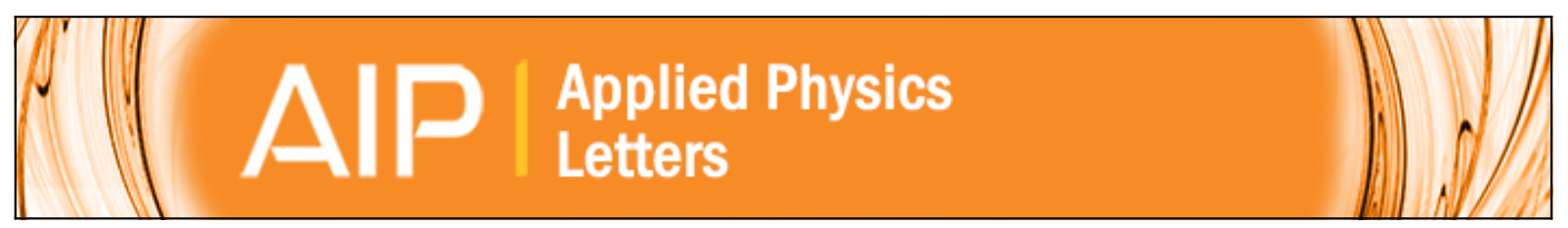

\title{
Trade-off between number of conductance states and variability of conductance change in $\mathrm{Pr} 0.7 \mathrm{Ca} 0.3 \mathrm{MnO}$-based synapse device
}

Daeseok Lee, Kibong Moon, Jaesung Park, Sangsu Park, and Hyunsang Hwang

Citation: Applied Physics Letters 106, 113701 (2015); doi: 10.1063/1.4915924

View online: http://dx.doi.org/10.1063/1.4915924

View Table of Contents: http://scitation.aip.org/content/aip/journal/apl/106/11?ver=pdfcov

Published by the AIP Publishing

\section{Articles you may be interested in}

Chemical-state-resolved depth profiles of $\mathrm{Al} / \mathrm{Pr} 0.7 \mathrm{Ca} 0.3 \mathrm{MnO} 3$ stacked structures for application in resistive switching devices

J. Appl. Phys. 114, 243711 (2013); 10.1063/1.4858381

Pressure dependence of resistivity and magnetoresistance in Pr-doped La0.7Ca0.3MnO3

J. Appl. Phys. 113, 17D721 (2013); 10.1063/1.4800679

Suppression of multi-level bipolar resistive switching in $\mathrm{Ag} / \mathrm{Pr} 0.7 \mathrm{Ca} 0.3 \mathrm{MnO} 3$ interfaces at low temperatures J. Appl. Phys. 112, 114511 (2012); 10.1063/1.4768843

Electroforming and endurance behavior of $\mathrm{Al} / \mathrm{Pr} 0.7 \mathrm{Ca} 0.3 \mathrm{MnO} 3 / \mathrm{Pt}$ devices

Appl. Phys. Lett. 99, 113506 (2011); 10.1063/1.3638059

Low temperature solution-processed graphene oxide/Pr0.7Ca0.3MnO3 based resistive-memory device Appl. Phys. Lett. 99, 042101 (2011); 10.1063/1.3617426

LIVE DEMO

The Basics of COMSOL in 18 Minutes

จ๐ С๐MSOL

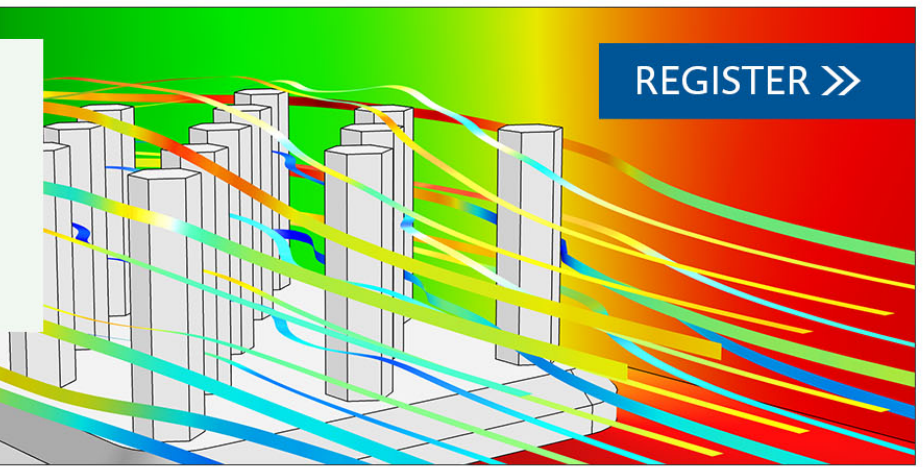




\title{
Trade-off between number of conductance states and variability of conductance change in $\operatorname{Pr}_{0.7} \mathrm{Ca}_{0.3} \mathrm{MnO}_{3}$-based synapse device
}

\author{
Daeseok Lee, ${ }^{1, a)}$ Kibong Moon, ${ }^{1, a)}$ Jaesung Park, ${ }^{1}$ Sangsu Park, ${ }^{2}$ \\ and Hyunsang Hwang ${ }^{1,2, b)}$ \\ ${ }^{1}$ Department of Materials Science and Engineering, Pohang University of Science and Technology \\ (POSTECH), 77 Cheongam-ro, Nam-gu, Pohang 790-784, South Korea \\ ${ }^{2}$ Department of Nanobio Material and Electronic, Gwangju Institute of Science and Technology (GIST), \\ 261 Cheomdan-gwagiro (Oryong-dong), Buk-gu, Gwangju 500-712, South Korea
}

(Received 15 January 2015; accepted 10 March 2015; published online 20 March 2015)

\begin{abstract}
The characteristics of $\operatorname{Pr}_{0.7} \mathrm{Ca}_{0.3} \mathrm{MnO}_{3}$ (PCMO)-based resistive change memory were evaluated as a synapse device for a emerging computing system inspired by a biological brain. The evaluated samples structured with various reactive top electrodes exhibited a dependence on the metal-oxide free energy. More conductance states which can improve the performance of the brain-inspired computing system were achieved in a sample having a low metal-oxide free energy. During the increase and decrease in the conductance, the low metal-oxide free energy also resulted in an asymmetric conductance change leading to degradation in the computational accuracy of the braininspired computing system. The results demonstrated a trade-off between the number of conductance states and the variation in the conductance change in the PCMO-based synapse device.
\end{abstract} (C) 2015 AIP Publishing LLC. [http://dx.doi.org/10.1063/1.4915924]

Recently, emerging computing systems inspired by a biological brain (brain-inspired system) have received considerable attention, and many studies have been conducted to implement these brain-inspired systems. ${ }^{1-3}$ For processing precisely defined data, the typical CMOS-based von Neumann architecture (typical computing system) is an ideal solution, but it is not suitable for problems including imprecisely defined data such as sensory data and big data. ${ }^{4}$ Even though we can improve the computational performance of a typical computing system to process imprecisely defined data, there are limitations with respect to power, area, and time. Thus, the brain-inspired system has been proposed as a strong candidate on the basis of its energy-efficient and error-tolerant computation of imprecisely defined data which can be utilized for unconventional applications such as a cognitive computing system and feature recognition system. $^{4-7}$ To realize the brain-inspired system, various approaches such as neural-network processes based on the von Neumann computing system, a CMOS-based hardware neural network (HNN) and a synapse-device-based HNN (S-HNN) have been proposed. ${ }^{4,73}$ Among these proposed approaches which have their associated advantages and disadvantages, the S-HNN can be the best solution in terms of the power, area, and time based on its massive parallelism, extremely high-density structure, power efficient operation, storage-combined computation, and robustness to computational faults. ${ }^{14}$ However, more studies are necessary from the synapse-device level to the system level.

With respect to the synapse device, various synapse devices such as resistive change memory (ReRAM), phase change memory, ferroelectric switches, and field-effecttransistor-based devices have been researched for the

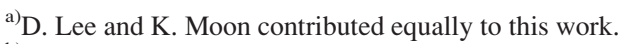
b) Author to whom correspondence should be addressed. Electronic mail:
hwanghs@postech.ac.kr
}

S-HNN. ${ }^{10-13,15-17}$ Even though each synapse device has its advantages, the synapse device needs a simple two-terminal structure for high density, low-power operation, an analogous conductance change, and reliable characteristics for the implementation of the biological-brain-like S-HNN. ${ }^{5}$ In these respects, ReRAM can be a promising candidate. In particular, $\operatorname{Pr}_{0.7} \mathrm{Ca}_{0.3} \mathrm{MnO}_{3}$ (PCMO)-based ReRAM can be utilized as a synapse device on the basis of its demonstrated reliable characteristics. ${ }^{11,12,18}$

According to previously reported studies, PCMO-based synapse devices (PCMO-synapse) exhibit spike-timingdependent plasticity (STDP) which is a change in the conductance of the PCMO-synapse by temporally modified pre- and post-neuron spikes, as shown in Fig. 1(a). ${ }^{11,12}$ When the pre-neuron spike is applied before the post-neuron spike, the conductance of the PCMO-synapse increases (potentiation). On the contrary, the conductance of the PCMO-synapse decreases when the post-neuron spike is applied before the preneuron spike (depression). These changes in the conductance of the PCMO-synapse can be considered as changes in the strength of the connection between pre- and post-neurons, which is a foundation of learning and memory in the biological brain. ${ }^{19}$ Considering the mechanism of computation in the biological brain, the PCMO-synapse can be employed as the storage-combined computational unit (synapse) in the S-HNN. ${ }^{20}$

Figs. 1(b)-1(d) show the simplified mechanism of the change in conductance during depression and potentiation. Fig. 1(b) shows the structure of the PCMO-synapse which is fabricated as a via-hole structure including a capping layer, a top electrode (TE), PCMO, and a bottom electrode (BE). The changes in the conductance result from a redox reaction between the reactive TE and the PCMO layer, as shown in Figs. 1(c) and 1(d). During depression, which is a process in which the conductance changes from high to low, a positive 

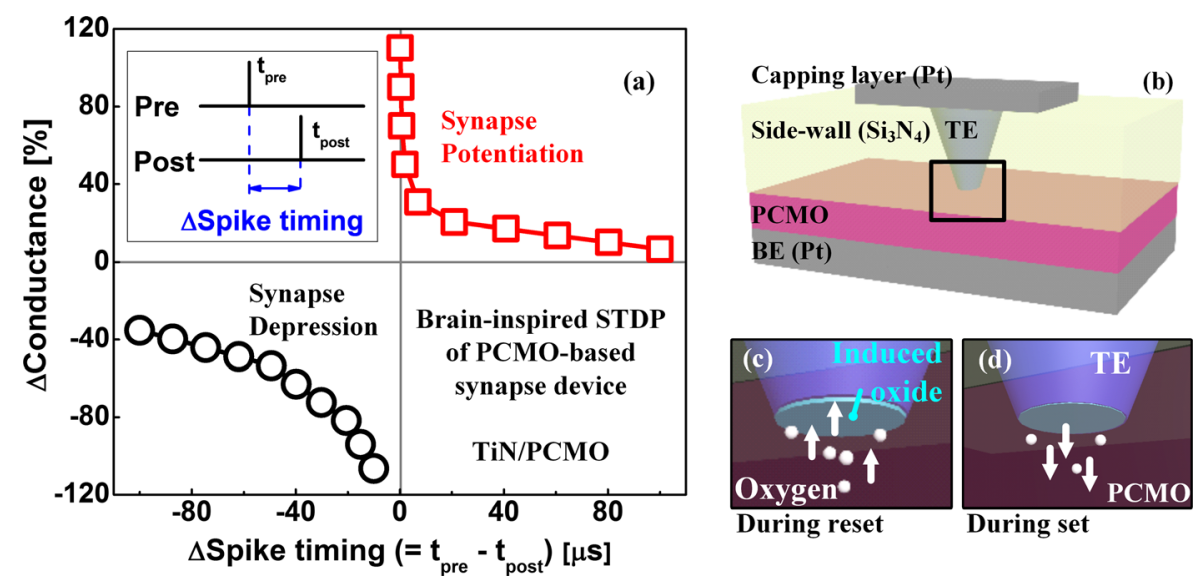

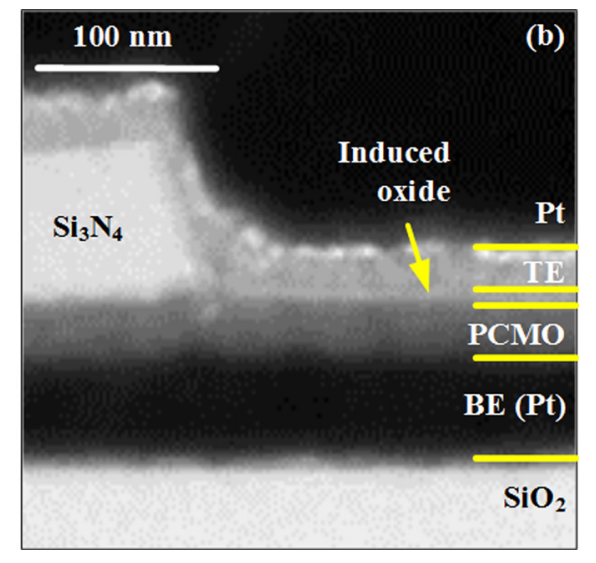

FIG. 1. (a) Spike-timing-dependent plasticity (STDP) of the evaluated PCMO-based synapse device (PCMOsynapse). ${ }^{11,12}$ The STDP allows for emulation of the brain-inspired computing systems with the PCMOsynapse. (b) Simple structure of the fabricated PCMO-synapses. (c) During depression (reset process), an induced oxide can be formed by oxygen absorption of reactive top electrode (TE). (d) In contrast to depression, the absorbed oxygens can move back to PCMO during potentiation (set process). This redox reaction of the TE leads to changes in the conductance of the PCMO-synapse.

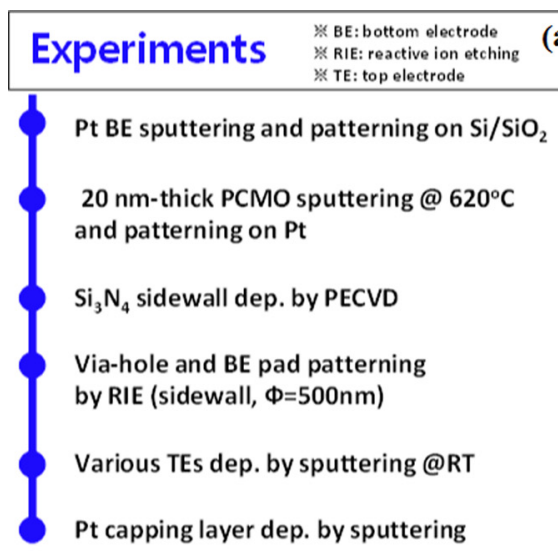

bias is applied to the reactive TE, and oxygen atoms in PCMO are attracted to the reactive TE. Then, these oxygen atoms react with the TE and form an induced oxide which can determine the total conductance of the PCMO-synapse. In contrast to depression, the oxygen atoms of the induced oxide move back to PCMO during potentiation, and the conductance is changed from low to high when a negative bias is applied to the TE. ${ }^{11,12,21-24}$ Considering an implementation of the S-HNN including a high-density and power-efficient synapse device, the redox reaction is the main key factor for improving the synaptic characteristics of the PCMO-synapse. In other words, the redox reaction between the reactive $\mathrm{TE}$ and the PCMO layer needs to be well-modified to obtain improved synaptic characteristics. Thus, in this study, we researched the dependence of the redox reaction on the synaptic characteristics by using various reactive TEs.

Fig. 2(a) shows the fabrication processes for the investigated samples: Pt/Ni/PCMO/Pt (Ni/PCMO), Pt/Ta/PCMO/Pt (Ta/PCMO), Pt/TiN/PCMO/Pt (TiN/PCMO), and Pt/Al/ $\mathrm{PCMO} / \mathrm{Pt}$ (Al/PCMO). Each sample was optimized by specific fabrication processes and bias conditions. ${ }^{11,12,18,21,22}$ Fig. 2(b) shows a cross-sectional transmission electron microscope (TEM) image of the initial state of the fabricated TiN/PCMO sample. During deposition of the reactive TE, an induced oxide was formed by oxygen absorption of the reactive TE. Figs. 3(a)-3(d) show the current versus voltage (I-V) characteristics of each sample for more than five DC cycles. All samples exhibited a negative set process, which is a resistance change from the high-resistance state (HRS) to the low-resistance state (LRS) under a set bias $\left(\mathrm{V}_{\text {set }}\right)$.
Moreover, under a positive reset bias $\left(\mathrm{V}_{\text {reset }}\right)$, the resistance changed from the LRS to the HRS (reset process). ${ }^{1,12}$ On the basis of the results from the I-V characteristics, the $\mathrm{Al} / \mathrm{PCMO}$ sample exhibited a larger operation voltage ratio $\left(=\mathrm{V}_{\text {set }} / \mathrm{V}_{\text {reset }}\right)$ and a larger on/off ratio (the ratio between the conductances in the LRS and HRS at the read voltage), as shown in Figs. 3(a)-3(e).

To investigate the synaptic characteristics, we evaluated the conductances of each sample during potentiation and depression by applying an identical pulse, as shown in Figs. 4(a)-4(d). For optimized operations, proper pulse bias conditions were utilized for each devices. From pulse number 0 to 50 , the conductance for potentiation was measured; then, the conductance for depression, which is plotted from pulse number 50 to 0 , was measured. In other words, the conductance was changed from the initial low conductance to the high conductance during potentiation (plotted as the black line with dots in Fig. 4). Subsequently, the conductance changed from a high conductance to a low conductance during depression (plotted as the red line with dots in Fig. 4). For the evaluated devices having little different conductance regions, normalized conductances (dividing by maximum conductance) were employed to compare the changes in the conductance $(\Delta G)$. In the ideal case, the $\Delta G$ should be the same during both potentiation and depression to obtain the same value of $\Delta \mathrm{G}$ per applied pulse (potentiation or depression pulse), as shown in Fig. 4(a). A variation in $\Delta \mathrm{G}$ can degrade the S-HNN because potentiation and depression can occur randomly during the learning process (information storage process) in the S-HNN. ${ }^{10,25}$ For example, if one 

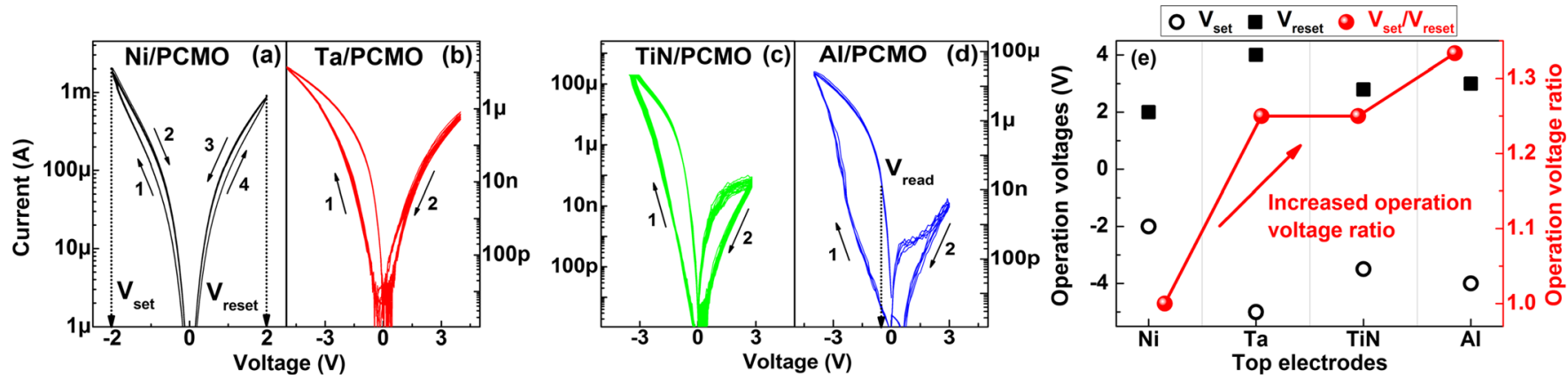

FIG. 3. (a)-(d) Current-voltage (I-V) curves of the fabricated samples. All samples exhibited counter-clockwise resistive switching including negative set and positive reset processes. Compared to the other samples, the Ni/PCMO sample exhibited a leaky and narrow resistive switching window, whereas the other samples exhibited wider on/off ratios with more asymmetric switching between the negative and positive bias regions. (e) Operation voltage ratio ( $=\mathrm{V}_{\text {set }} /$ $\mathrm{V}_{\text {reset }}$ ) of the evaluated samples. The Ni/PCMO sample exhibited the same operation voltages, while the other samples have asymmetric operation voltages. Considering the activation energy during depression and potentiation, these asymmetric switching characteristics can lead to asymmetric synaptic characteristics.
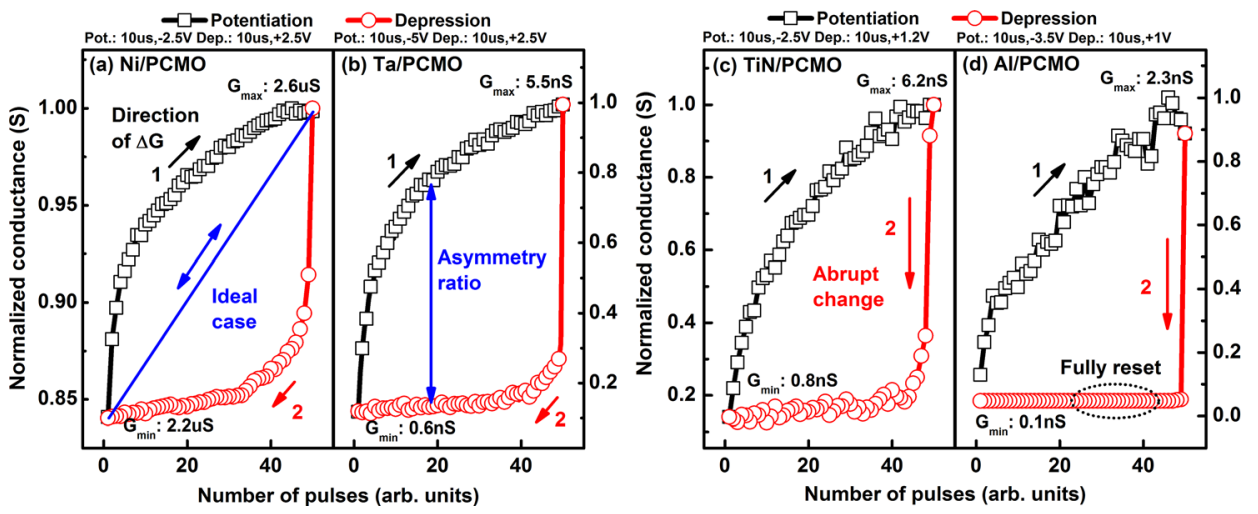

FIG. 4. (a)-(d) The PCMO-synapses exhibited various conductance changes $(\Delta \mathrm{G})$ which are the most important synaptic characteristic. In the ideal case, $\Delta \mathrm{G}$ is the same during potentiation and depression [described in (a)]. However, the PCMO-synapses exhibited asymmetric values of $\Delta \mathrm{G}$ which can lead to a degradation in the performance of the S-HNN. ${ }^{10,25}$ The asymmetry of $\Delta \mathrm{G}$ can be defined as an asymmetry ratio (conductance ratio between potentiation and depression), as shown in the (b).
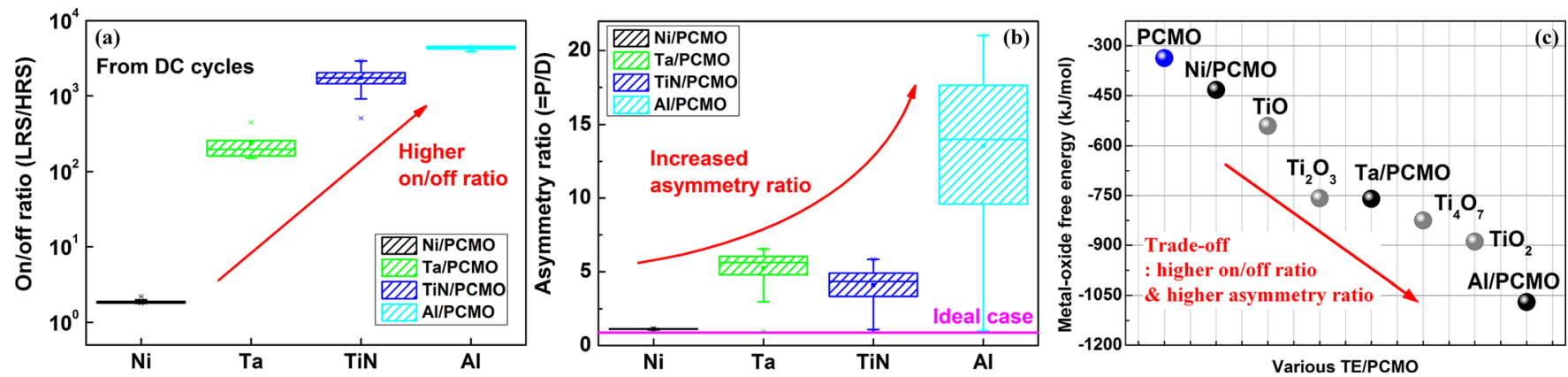

FIG. 5. (a) On/off ratio (ratio between the conductances in the LRS and HRS at the read voltage) for various TEs. From Ni to Ta to TiN to Al, the on/off ratio and (b) asymmetry ratio increased. The Al/PCMO sample exhibited the largest on/off ratio, leading to a high number of conductance states and the largest asymmetry ratio that can cause errors in computation in the S-HNN. ${ }^{10,25}$ (c) On the basis of the Gibbs free energy of the induced oxides, ${ }^{27,28}$ these results can be considered as a trade-off between two important requirements of the synapse device: the number of conductance states and the similarity of the value of $\Delta \mathrm{G}$. For higher performance of the S-HNN, the synapse devices need to satisfy both requirements (a large number of conductance states and stable $\Delta \mathrm{G}$ ). ${ }^{10,25}$

potentiation pulse and one depression pulse were applied sequentially, the final conductance needs to be similar to the initial conductance. Even though the S-HNN has errortolerant computation, a larger variation in $\Delta \mathrm{G}$ can lead to a degradation in the computation of the S-HNN. ${ }^{10,25}$ In this respect, the similarity of $\Delta \mathrm{G}$ during potentiation and depression is an important requirement for a synapse device. To evaluate the similarity of $\Delta \mathrm{G}$, we defined the asymmetry ratio, which is the conductance ratio between potentiation and depression, as shown in Fig. 4(b).

Fig. 5 shows the results for the TE dependence: the tendencies of the on/off ratio and the asymmetry ratio. The on/off ratio in Fig. 5(a) increased when the TE was changed from $\mathrm{Ni}$ to $\mathrm{Ta}$ to $\mathrm{TiN}$ to $\mathrm{Al}$, which means that a larger number of conductance states can be obtained in the Al/PCMO sample. This is also an important synaptic characteristic because a larger number of conductance states lead to higher performance of the S-HNN. ${ }^{5,10,25}$ Among the evaluated samples in Fig. 5(b), the Al/PCMO sample exhibited the highest asymmetry ratio, which can lead to a degradation in computation in the S-HNN. These results (the highest ratio and asymmetry ratio) can be explained by the Gibbs free energy of the induced oxide (metal-oxide free energy). ${ }^{26-28}$ The free energy of $\mathrm{NiO}_{\mathrm{x}}$ induced in the $\mathrm{Ni} / \mathrm{PCMO}$ sample is similar to that of 
PCMO, whereas $\mathrm{AlO}_{\mathrm{x}}$ of the $\mathrm{Al} / \mathrm{PCMO}$ sample has a smaller metal-oxide free energy than that of PCMO, as shown in Fig. 5(c). This means that the induced $\mathrm{AlO}_{\mathrm{x}}$ is more stable than PCMO in the Al/PCMO sample. In other words, the activation energy for the oxidation of $\mathrm{Al}$ (depression or reset) is smaller than that for the reduction of $\mathrm{Al}$ (potentiation or set). ${ }^{27,28}$ Thus, the Al/PCMO sample exhibited a smaller value for $\mathrm{V}_{\text {reset }}$ than $\mathrm{V}_{\text {set }}$, and the operation voltage ratio $\left(=\mathrm{V}_{\text {set }} / \mathrm{V}_{\text {reset }}\right)$ was higher than the others [Figs. 3(e) and 4]. In addition, the smaller activation energy for the oxidation of $\mathrm{Al}$ can lead to an abrupt $\Delta \mathrm{G}$ during the depression, which is the origin of the higher asymmetry ratio, as shown in Fig. 4(d). For TiN and Ta, TiN TE can form various titanium oxides such as $\mathrm{TiO}, \mathrm{Ti}_{2} \mathrm{O}_{3}, \mathrm{Ti}_{4} \mathrm{O}_{7}$, and $\mathrm{TiO}_{2}$. Compare to the metaloxide free energy of $\mathrm{Ta} / \mathrm{PCMO}$ sample, $\mathrm{TiO}$ and $\mathrm{TiO}_{2}$ have difference in the metal-oxide free energy, but $\mathrm{Ti}_{2} \mathrm{O}_{3}$ and $\mathrm{Ti}_{4} \mathrm{O}_{7}$ have similar metal-oxide free energy. Thus, the $\mathrm{Ta} / \mathrm{PCMO}$ and TiN/PCMO samples exhibited similar tendencies, as shown in Fig. 5. These results mean that the PCMOsynapses have a trade-off between the number of conductance states and the similarity in the values of $\Delta \mathrm{G}$.

For the implementation of an S-HNN, the synaptic characteristics of a PCMO-synapse were evaluated by using various TEs. On the basis of the metal-oxide free energy, the evaluated samples exhibited a trade-off between the on/off ratio and the asymmetry ratio. To obtain a more proper PCMO-synapse for the S-HNN, the metal-oxide free energy of the TEs should be carefully considered; a lower metaloxide free energy can lead to a high on/off ratio but can increase the asymmetry ratio.

This research was supported by the Pioneer Research Center Program through the National Research Foundation of Korea funded by the Ministry of Science, ICT \& Future Planning (2012-0009460).

${ }^{1}$ B. Widrow and M. E. Hoff, IRE WESCON Convention Record, 1960.

${ }^{2}$ K. Steinbuch and M. Peter, U.S. patent 3,286,238 (1966).

${ }^{3}$ R. Stanford and I. M. Ovshinsky, Mater. Res. Bull. 5(8), 681 (1970).

${ }^{4}$ P. A. Merolla, J. V. Arthur, R. Alvarez-Icaza, A. S. Cassidy, J. Sawada, F. Akopyan, B. L. Jackson, N. Imam, C. Guo, and Y. Nakamura, Science 345(6197), 668 (2014).

${ }^{5}$ D. Kuzum, S. Yu, and H. S. Philip Wong, Nanotechnology 24(38), 382001 (2013).
${ }^{6}$ J. Von Neumann, IEEE Ann. Hist. Comput. 15(4), 27 (1993).

${ }^{7}$ D. Ferrucci, E. Brown, J. Chu-Carroll, J. Fan, D. Gondek, A. A. Kalyanpur, A. Lally, J. William Murdock, E. Nyberg, and J. Prager, AI Mag. 31(3), 59 (2010).

${ }^{8}$ G. Vishnu Varatkar, S. Narayanan, N. R. Shanbhag, and D. L. Jones, IEEE Trans. Very Large Scale Integr. (VLSI) Syst. 18(10), 1421 (2010).

${ }^{9} \mathrm{~K}$. Ho Lee, K. Jin Jang, A. Shoeb, and N. Verma, paper presented at the Engineering in Medicine and Biology Society, EMBC, Annual International Conference of the IEEE, 2011.

${ }^{10}$ G. W. Burr, R. M. Shelby, C. di Nolfo, J. W. Jang, R. S. Shenoy, P. Narayanan, K. Virwani, E. U. Giacometti, B. Kurdi, and H. Hwang, paper presented at the IEEE International Electron Devices Meeting (IEDM), 2014.

${ }^{11}$ S. Park, H. Kim, M. Choo, J. Noh, A. Sheri, S. Jung, K. Seo, J. Park, S. Kim, and W. Lee, paper presented at the IEEE International Electron Devices Meeting (IEDM), 2012.

${ }^{12}$ S. Park, A. Sheri, J. Kim, J. Noh, J. Jang, M. Jeon, B. Lee, B. R. Lee, B. $\mathrm{H}$. Lee, and H. Hwang, paper presented at the IEEE International Electron Devices Meeting (IEDM), 2013.

${ }^{13}$ M. Suri, O. Bichler, D. Querlioz, O. Cueto, L. Perniola, V. Sousa, D. Vuillaume, C. Gamrat, and B. DeSalvo, paper presented at the IEEE International Electron Devices Meeting (IEDM), 2011.

${ }^{14}$ E. Guizzo, IEEE Spectrum 17 (2011).

${ }^{15}$ S.-M. Yoon, E. Tokumitsu, and H. Ishiwara, IEEE Electron Device Lett. 20(5), 229 (1999).

${ }^{16}$ A. Chanthbouala, V. Garcia, R. O. Cherifi, K. Bouzehouane, S. Fusil, X. Moya, S. Xavier, H. Yamada, C. Deranlot, and N. D. Mathur, Nat. Mater. 11(10), 860 (2012).

${ }^{17}$ G. Agnus, W. Zhao, V. Derycke, A. Filoramo, Y. Lhuillier, S. Lenfant, D. Vuillaume, C. Gamrat, and J. Philippe Bourgoin, Adv. Mater. 22(6), 702 (2010).

${ }^{18}$ D.-J. Seong, M. Hassan, H. Choi, J. Lee, J. Yoon, J.-B. Park, W. Lee, M.S. Oh, and H. Hwang, IEEE Electron Device Lett. 30(9), 919 (2009).

${ }^{19}$ H. Markram, J. Lübke, M. Frotscher, and B. Sakmann, Science 275(5297), 213 (1997).

${ }^{20}$ D. Olding Hebb, The Organization of Behavior: A Neuropsychological Theory (Wiley, 1949).

${ }^{21}$ S. Park, J. Noh, M.-1. Choo, A. Muqeem Sheri, M. Chang, Y.-B. Kim, C. Jung Kim, M. Jeon, B.-G. Lee, and B. Hun Lee, Nanotechnology 24(38), 384009 (2013).

${ }^{22}$ S. Park, M. Siddik, J. Noh, D. Lee, J. Woo, B. Hun Lee, and H. Hwang, Semicond. Sci. Technol. 29(10), 104006 (2014).

${ }^{23}$ Z. L. Liao, Z. Z. Wang, Y. Meng, Z. Y. Liu, P. Gao, J. L. Gang, H. W. Zhao, X. J. Liang, X. D. Bai, and D. M. Chen, Appl. Phys. Lett. 94(25), 253503 (2009).

${ }^{24}$ T. Nakamura, K. Homma, and K. Tachibana, Nanoscale Res. Lett. 8(1), 1 (2013).

${ }^{25}$ J.-W. Jang, S. Park, Y.-H. Jeong, and H. Hwang, paper presented at the IEEE International Symposium on Circuits and Systems (ISCAS), 2014.

${ }^{26}$ H.-G. Lee, Chemical Thermodynamics for Metals and Materials (Imperial College Press, 1999).

${ }^{27}$ G. Valentinovic Samsonov, The Oxide Handbook (Ifi/Plenum, 1982).

${ }^{28}$ S. Shirasaki, H. Yamamura, H. Haneda, K. Kakegawa, and J. Moori, J. Chem. Phys. 73(9), 4640 (1980). 